Bull. Korean Math. Soc. 48 (2011), No. 3, pp. 575-584

DOI 10.4134/BKMS.2011.48.3.575

\title{
SOME NOTES ON ISHIKAWA ITERATION FOR MULTI-VALUED MAPPINGS
}

\author{
Yisheng Song And YeOL Je Cho
}

\begin{abstract}
In Shahzad and Zegeye [Nonlinear Anal. 71 (2009), no. 3-4, 838-844], the authors introduced several Ishikawa iterative schemes for fixed points of multi-valued mappings in Banach spaces, and proved some strong convergence theorems by using their iterations. In their proofs of the main results, it seems reasonable and simpler to prove for the iteration $\left\{x_{n}\right\}$ to be a Cauchy sequence. In this paper, we modify and improve the proofs of the main results given by Shahzad and Zegeye. Two concrete examples also are given.
\end{abstract}

\section{Introduction}

Throughout this paper, let $E$ be a Banach space with the norm $\|\cdot\|$ and $E^{*}$ be the dual spaces of $E$. Let $\mathbb{N}$ denote the set of all positive integers.

Let $K$ be a nonempty subset of $E$. The set $K$ is said to be proximinal if, for any $x \in E$, there exists an element $y \in K$ such that $\|x-y\|=d(x, K)$, where $d(x, K)=\inf \{\|x-y\| ; y \in K\}$. We shall denote $C B(K), C(K)$ and $P(K)$ by the families of nonempty closed and bounded subsets, nonempty compact subsets and nonempty proximinal bounded subsets of $K$, respectively. Let $H$ be Hausdorff metric on $C B(E)$ defined by

$$
H(A, B)=\max \left\{\sup _{x \in A} d(x, B), \sup _{x \in B} d(x, A)\right\}, \quad \forall A, B \in C B(E) .
$$

A multi-valued mapping $T: K \rightarrow C B(K)$ is said to be nonexpansive (resp., contractive) if

$$
\begin{gathered}
H(T x, T y)\|\leq\| x-y \|, \quad \forall x, y \in K, \\
\text { (resp., } H(T x, T y) \leq k\|x-y\| \text { for some } k \in(0,1)) .
\end{gathered}
$$

Received September 24, 2009; Revised March 22, 2011.

2010 Mathematics Subject Classification. 47H09, 47H10, 47J25, 47J05.

Key words and phrases. multi-valued mapping, strong convergence, Ishikawa iteration.

The first author was supported by the National Natural Science Foundation of P. R. China (11071279), and by the Research Programs of Basic and Cutting-edge Technology of Henan Province (102300410012), and by the Natural Science Research Projects (Basic Research Project) of Education Department of Henan Province (2009B110011, 2009B110001).

The second author was supported by the Korea Research Foundation Grant funded by the Korean Government (KRF-2008-313-C00050). 
A point $x$ is called a fixed point of $T$ if $x \in T x$. Here $F(T)$ stands for the set of fixed points of a mapping $T$. The mapping $T: K \rightarrow C B(K)$ is said to be quasi-nonexpansive ([3]) if $F(T) \neq \emptyset$ and

$$
H(T x, T p)\|\leq\| x-p \|, \quad \forall x \in K, p \in F(T) .
$$

It is clear that every nonexpansive multi-valued mapping $T$ with $F(T) \neq \emptyset$ is quasi-nonexpansive. But there exist quasi-nonexpansive mappings that are not nonexpansive (see [4] and [3]).

The mapping $T: K \rightarrow C B(K)$ is said to Condition $(I)$ if there is a nondecreasing function $f:[0,+\infty) \rightarrow[0,+\infty)$ with $f(0)=0$ and $f(r)>0$ for all $r>0$ such that

$$
d(x, T x) \geq f(d(x, F(T)), \quad \forall x \in K .
$$

The mapping $T: K \rightarrow C B(K)$ is said to be hemi-compact if, for any sequence $\left\{x_{n}\right\}$ in $K$ such that $\lim _{n \rightarrow \infty} d\left(x_{n}, T x_{n}\right)=0$, there exists a subsequence $\left\{x_{n_{k}}\right\}$ of $\left\{x_{n}\right\}$ such that $\lim _{k \rightarrow \infty} x_{n_{k}}=x \in K$.

Sastry and Babu [2] defined the Ishikawa iterative scheme for multi-valued mappings as follows:

(A) Let $T: K \rightarrow P(K)$ be a multi-valued mapping and fix $p \in F(T)$.

$$
\left\{\begin{array}{l}
x_{0} \in K, \\
y_{n}=\left(1-\beta_{n}\right) x_{n}+\beta_{n} z_{n}, \quad \forall n \geq 0,
\end{array}\right.
$$

where $\left\{\beta_{n}\right\}$ is a sequence in $[0,1]$ and $z_{n} \in T x_{n}$ such that $\left\|z_{n}-p\right\|=d\left(p, T x_{n}\right)$, and

$$
\left\{\begin{array}{l}
x_{0} \in K, \\
x_{n+1}=\left(1-\alpha_{n}\right) x_{n}+\alpha_{n} z_{n}^{\prime}, \quad \forall n \geq 0,
\end{array}\right.
$$

where $\left\{\alpha_{n}\right\}$ is a sequence in $[0,1]$ and $z_{n}^{\prime} \in T y_{n}$ such that $\left\|z_{n}^{\prime}-p\right\|=d\left(p, T y_{n}\right)$.

They also proved the strong convergence of the above Ishikawa iterative scheme for a multi-valued nonexpansive mapping $T$ with a fixed point $p$ under certain conditions in a Hilbert space.

Recently, Panyanak [1] extended the results of Sastry and Babu [2] to a uniformly convex Banach space, and also modified the above Ishikawa iterative scheme as follows:

(B) Let $T: K \rightarrow P(K)$ be a multi-valued mapping.

$$
\left\{\begin{array}{l}
x_{0} \in K, \\
y_{n}=\left(1-\beta_{n}\right) x_{n}+\beta_{n} z_{n}, \quad \forall n \geq 0,
\end{array}\right.
$$

where $\left\{\beta_{n}\right\}$ is a sequence in $[0,1], z_{n} \in T x_{n}$ and $u_{n} \in T x_{n}$ are such that $\left\|z_{n}-u_{n}\right\|=d\left(u_{n}, T x_{n}\right)$ and $\left\|x_{n}-u_{n}\right\|=d\left(x_{n}, F(T)\right)$, respectively, and

$$
\left\{\begin{array}{l}
x_{0} \in K, \\
x_{n+1}=\left(1-\alpha_{n}\right) x_{n}+\alpha_{n} z_{n}^{\prime}, \quad \forall n \geq 0,
\end{array}\right.
$$


where $\left\{\alpha_{n}\right\}$ is a sequence in $[0,1], z_{n}^{\prime} \in T x_{n}$ and $v_{n} \in T x_{n}$ are such that $\left\|z_{n}^{\prime}-v_{n}\right\|=d\left(v_{n}, T x_{n}\right)$ and $\left\|y_{n}-v_{n}\right\|=d\left(y_{n}, F(T)\right)$, respectively.

Very recently, Song and Wang $[5,6]$ noted that there was a gap in the proofs of [1, Theorem 3.1] and [2, Theorem 5]. Because the iteration $\left\{x_{n}\right\}$ depends on the point $p$ as well as the mapping $T$. If $p, q \in F(T)$ and $p \neq q$, then the iteration $\left\{x_{n}\right\}$ defined by the point $q$ is different from that defined by the point $p$. Therefore, the conclusion of [1, Theorem 3.1] and [2, Theorem 5] are ambiguous. Thus they solved/revised the gap by means of the following Ishikawa iterative scheme:

(C) Let $T: K \rightarrow C B(K)$ be a multi-valued mapping, $\beta_{n} \in[0,1]$ and $\alpha_{n} \in[0,1]$. The Ishikawa iterative scheme $\left\{x_{n}\right\}$ is defined by

$$
\left\{\begin{array}{l}
x_{0} \in K, \\
y_{n}=\left(1-\beta_{n}\right) x_{n}+\beta_{n} z_{n}, \\
x_{n+1}=\left(1-\alpha_{n}\right) x_{n}+\alpha_{n} z_{n}^{\prime}, \quad \forall n \geq 0,
\end{array}\right.
$$

where $z_{n} \in T x_{n}$ and $z_{n}^{\prime} \in T y_{n}$ are such that $\left\|z_{n}-z_{n}^{\prime}\right\| \leq H\left(T x_{n}, T y_{n}\right)+\gamma_{n}$ and $\left\|z_{n+1}-z_{n}^{\prime}\right\| \leq H\left(T x_{n+1}, T y_{n}\right)+\gamma_{n}$, respectively, and $\gamma_{n} \in(0,+\infty)$ is such that $\lim _{n \rightarrow \infty} \gamma_{n}=0$.

They also proved the following strong convergence theorems for the Ishikawa iterative scheme in $(C)$ :

Theorem SW1 ([5, Theorem 1]). Let $K$ be a nonempty compact convex subset of a uniformly convex Banach space E. Suppose that $T: K \rightarrow C B(K)$ is a multi-valued nonexpansive mapping and $F(T) \neq \emptyset$ satisfying $T(y)=\{y\}$ for any $y \in F(T)$. Assume that the sequences $\left\{\alpha_{n}\right\}$ and $\left\{\beta_{n}\right\}$ in $[0,1)$ satisfy the following conditions:

(i) $\lim _{n \rightarrow \infty} \beta_{n}=0$;

(ii) $\sum_{n=0}^{\infty} \alpha_{n} \beta_{n}=\infty$.

Then the Ishikawa iterative scheme defined in $(C)$ converges to a fixed point of $T$.

Theorem SW2 ([5, Theorem 2]). Let $K$ be a nonempty closed convex subset of a uniformly convex Banach space E. Suppose that $T: K \rightarrow C B(K)$ is a multi-valued nonexpansive mapping that satisfies Condition (I). Assume that $F(T) \neq \emptyset$ satisfying $T(y)=\{y\}$ for any $y \in F(T)$ and $\alpha_{n}, \beta_{n} \in[a, b] \subset(0,1)$. Then the Ishikawa iterative scheme $\left\{x_{n}\right\}$ defined in $(C)$ converges to a fixed point of $T$.

More recently, Shahzad and Zegeye [4] modified the Ishikawa iterative scheme $\left\{x_{n}\right\}$ defined in $(C)$ and extended Theorem SW2 to a multi-valued quasinonexpansive mapping as follows: 
(D) Let $T: K \rightarrow C B(K)$ be a multi-valued mapping, $\beta_{n} \in[0,1]$ and $\alpha_{n} \in[0,1]$. The Ishikawa iterative scheme $\left\{x_{n}\right\}$ is defined by

$$
\left\{\begin{array}{l}
x_{0} \in K \\
y_{n}=\left(1-\beta_{n}\right) x_{n}+\beta_{n} z_{n}, \\
x_{n+1}=\left(1-\alpha_{n}\right) x_{n}+\alpha_{n} z_{n}^{\prime}, \quad \forall n \geq 0
\end{array}\right.
$$

where $z_{n} \in T x_{n}$ and $z_{n}^{\prime} \in T y_{n}$.

They also proved the following theorems:

Theorem SZ1 ([4, Theorem 2.3]). Let $K$ be a nonempty closed convex subset of a uniformly convex Banach space E. Suppose that $T: K \rightarrow C B(K)$ is a multi-valued quasi-nonexpansive mapping that satisfies Condition (I). Assume that $F(T) \neq \emptyset$ satisfying $T(y)=\{y\}$ for any $y \in F(T)$ and $\alpha_{n}, \beta_{n} \in[a, b] \subset$ $(0,1)$. Then the Ishikawa iterative scheme $\left\{x_{n}\right\}$ defined in $(D)$ converges to a fixed point of $T$.

Theorem SZ2 ([4, Theorem 2.5]). Let $K$ be a nonempty closed convex subset of a uniformly convex Banach space E. Suppose that $T: K \rightarrow C B(K)$ is a multi-valued quasi-nonexpansive mapping and $F(T) \neq \emptyset$ satisfying $T(y)=\{y\}$ for any $y \in F(T)$. Assume that $T$ is hemi-compact and continuous and the sequences $\left\{\alpha_{n}\right\},\left\{\beta_{n}\right\}$ in $[0,1)$ satisfy the following conditions:

(ii) $\lim _{n \rightarrow \infty} \beta_{n}=0$;

(iii) $\sum_{n=0}^{\infty} \alpha_{n} \beta_{n}=\infty$.

Then the Ishikawa iterative scheme $\left\{x_{n}\right\}$ defined in $(D)$ converges to a fixed point of $T$.

For removing the rigid restriction on $F(T)$, that is, $T(y)=\{y\}$ for any $y \in F(T)$, Shahzad and Zegeye [4] introduced the following iterative scheme:

(E) Let $T: K \rightarrow P(K)$ be a multi-valued mapping and $P_{T}(x)=\{y \in K$ : $\|x-y\|=d(x, T x)\}$. The Ishikawa iterative scheme $\left\{x_{n}\right\}$ is defined by

$$
\left\{\begin{array}{l}
x_{0} \in K \\
y_{n}=\left(1-\beta_{n}\right) x_{n}+\beta_{n} z_{n}, \\
x_{n+1}=\left(1-\alpha_{n}\right) x_{n}+\alpha_{n} z_{n}^{\prime}, \quad \forall n \geq 0,
\end{array}\right.
$$

where $\alpha_{n}, \beta_{n} \in[0,1], z_{n} \in P_{T}\left(x_{n}\right)$ and $z_{n}^{\prime} \in P_{T}\left(y_{n}\right)$.

They also proved the following theorems:

Theorem SZ3 ([4, Theorem 2.7]). Let $K$ be a nonempty closed convex subset of a uniformly convex Banach space $E$. Suppose that $T: K \rightarrow P(K)$ is a multi-valued mapping with $F(T) \neq \emptyset$ and $P_{T}$ is nonexpansive. Assume that $T$ satisfies Condition $(I)$ and $\alpha_{n}, \beta_{n} \in[a, b] \subset(0,1)$. Then the Ishikawa iterative scheme $\left\{x_{n}\right\}$ defined in $(E)$ converges to a fixed point of $T$. 
Theorem SZ4 ([4, Theorem 2.8]). Let $K$ be a nonempty closed convex subset of a uniformly convex Banach space E. Suppose that $T: K \rightarrow P(K)$ is a multi-valued mapping with $F(T) \neq \emptyset$ and $P_{T}$ is nonexpansive. Assume that $T$ is hemi-compact and the sequences $\left\{\alpha_{n}\right\},\left\{\beta_{n}\right\}$ in $[0,1)$ satisfy the following conditions:

(i) $\lim _{n \rightarrow \infty} \beta_{n}=0$;

(ii) $\sum_{n=0}^{\infty} \alpha_{n} \beta_{n}=\infty$.

Then the Ishikawa iterative scheme $\left\{x_{n}\right\}$ defined in $(E)$ converges to a fixed point of $T$.

In the proofs of Theorem SW2, SZ1, SZ3 as well as Theorem 3.8 of Panyanak [1], by taking a convergent subsequence $\left\{x_{n_{k}}\right\}$ of the given sequence $\left\{x_{n}\right\}$ in $F(T)$, they proved some strong convergence theorems for the Mann and Ishikawa iterative scheme $\left\{x_{n}\right\}$. However, it seems more reasonable and simpler to use a direct proof for a Cauchy sequence of the iterative scheme $\left\{x_{n}\right\}$, which is one of the aims of this paper. We extend and improve the main results of Shahzad and Zegeye [4] and also give two concrete examples.

\section{Main results}

In this section, we first give a direct proof for a Cauchy sequence of the iterative secheme $\left\{x_{n}\right\}$ defined in Theorems SW2, SZ1, SZ3 as well as Theorem 3.8 of Panyanak [1].

Proof of Theorem SZ1. Using the same proof technique of Theorem SZ1 (also see the proof of Theorems SW1, SZ3 and Theorem 3.1 of Panyanak [1]), we can obtain the following:

(i) $\left\|x_{n+1}-p\right\| \leq\left\|x_{n}-p\right\|$ for each $p \in F(T)$;

(ii) for each $p \in F(T), \lim _{n \rightarrow \infty}\left\|x_{n}-p\right\|$ exists;

(iii) $\lim _{n \rightarrow \infty} d\left(x_{n}, T x_{n}\right)=0$.

Since $T$ satisfies Condition $(I)$, we have

$$
\lim _{n \rightarrow \infty} d\left(x_{n}, F(T)\right)=0 .
$$

Now, we claim that $\left\{x_{n}\right\}$ is a Cauchy sequence in $K$. In fact, let $m, n \in \mathbb{N}$. Without loss of generality, we assume that $m>n$. Then it follows that

$$
\left\|x_{m}-p\right\| \leq\left\|x_{n}-p\right\|, \quad \forall p \in F(T) .
$$

Thus we have

$$
\left\|x_{m}-x_{n}\right\| \leq\left\|x_{m}-p\right\|+\left\|p-x_{n}\right\| \leq 2\left\|x_{n}-p\right\|
$$

and so, taking the infimum for all $p \in F(T)$, we have

$$
\left\|x_{m}-x_{n}\right\| \leq 2 d\left(x_{n}, F(T)\right) \rightarrow 0(n \rightarrow \infty) .
$$


That is,

$$
\left\|x_{m}-x_{n}\right\| \rightarrow 0(m, n \rightarrow \infty) .
$$

This shows that $\left\{x_{n}\right\}$ is a Cauchy sequence in $K$ and so it converges to a point $x^{*} \in K$.

Next, we show that $x^{*} \in F(T)$. Indeed, by $d\left(x_{n}, F(T)\right)=\inf _{y \in F(T)}\left\|x_{n}-y\right\|$, for each $\varepsilon>0$, there exists $p_{n}(\varepsilon) \in F(T)$ such that

$$
\left\|x_{n}-p_{n}(\varepsilon)\right\| \leq d\left(x_{n}, F(T)\right)+\frac{\varepsilon}{2} .
$$

This implies $\lim _{n \rightarrow \infty}\left\|x_{n}-p_{n}(\varepsilon)\right\| \leq \frac{\varepsilon}{2}$. From $\left\|p_{n}(\varepsilon)-x^{*}\right\| \leq\left\|x_{n}-p_{n}(\varepsilon)\right\|+\| x_{n}-$ $x^{*} \|$, it follows that

$$
\limsup _{n \rightarrow \infty}\left\|p_{n}(\varepsilon)-x^{*}\right\| \leq \frac{\varepsilon}{2}
$$

Since

$$
\begin{aligned}
d\left(x^{*}, T x^{*}\right) & \leq\left\|x^{*}-p_{n}(\varepsilon)\right\|+d\left(p_{n}(\varepsilon), T x^{*}\right) \\
& \leq\left\|x^{*}-p_{n}(\varepsilon)\right\|+H\left(T\left(p_{n}(\varepsilon)\right), T x^{*}\right) \\
& \leq 2\left\|p_{n}(\varepsilon)-x^{*}\right\| .
\end{aligned}
$$

Then we have $d\left(x^{*}, T x^{*}\right) \leq 2 \limsup _{n \rightarrow \infty}\left\|p_{n}(\varepsilon)-x^{*}\right\| \leq \varepsilon$. Since $\varepsilon$ is arbitrary, then $d\left(x^{*}, T x^{*}\right)=0$. So $x^{*} \in F(T)$. This complete the proof.

Lemma 1. Let $T: K \rightarrow P(K)$ be a multi-valued mapping and $P_{T}(x)=\{y \in$ $K ;\|x-y\|=d(x, T x)\}$. Then the following are equivalent:

(1) $x \in F(T)$, that is, $x \in T x$;

(2) $P_{T}(x)=\{x\}$, that is, $x=y$ for each $y \in P_{T}(x)$;

(3) $x \in F\left(P_{T}\right)$, that is, $x \in P_{T}(x)$.

Further, $F(T)=F\left(P_{T}\right)$.

Proof. (1) implies (2). Since $x \in T x$, then $d(x, T x)=0$. Therefore, for any $y \in P_{T}(x),\|x-y\|=d(x, T x)=0$ and so $x=y$. That is, $P_{T}(x)=\{x\}$.

Clearly, (2) implies (3).

(3) implies (1). Since $x \in P_{T}(x), d(x, T x)=\|x-x\|=0$ and so $x \in T x$ by the closedness of $T x$.

Let $T: K \rightarrow P(K)$ be a multi-valued mapping and $P_{T}(x)=\{y \in K ; \| x-$ $y \|=d(x, T x)\}$. The Ishikawa iterative scheme $\left\{x_{n}\right\}$ is defined by

$$
\left\{\begin{array}{l}
x_{0} \in K, \\
y_{n}=\left(1-\beta_{n}\right) x_{n}+\beta_{n} z_{n}, \\
x_{n+1}=\left(1-\alpha_{n}\right) x_{n}+\alpha_{n} z_{n}^{\prime}, \quad \forall n \geq 0,
\end{array}\right.
$$

where $\left\{\alpha_{n}\right\},\left\{\beta_{n}\right\}$ are sequences in $[0,1], z_{n} \in P_{T}\left(x_{n}\right)$ and $z_{n}^{\prime} \in P_{T}\left(y_{n}\right)$.

Following Theorem SZ1 (or Theorem SW2) together with Lemma 1, we have the following corollary which contains Theorem SZ3 as a special case: 
Theorem 2. Let $K$ be a nonempty closed convex subset of a uniformly convex Banach space E. Suppose that $T: K \rightarrow P(K)$ is a multi-valued mapping with $F(T) \neq \emptyset$ and $P_{T}$ is quasi-nonexpansive. Assume that $T$ satisfies Condition $(I)$ and $\alpha_{n}, \beta_{n} \in[a, b] \subset(0,1)$. Then the Ishikawa iterative scheme $\left\{x_{n}\right\}$ defined by $(*)$ converges to a fixed point of $T$.

Proof. It follows from Lemma 1 that

$$
F(T)=F\left(P_{T}\right) \neq \emptyset, \quad P_{T}(x)=\{x\}, \quad \forall x \in F\left(P_{T}\right) .
$$

It follows from the definition of $P_{T}$ that $P_{T}(x) \subset T(x)$, which means that

$$
d(x, T x)=\inf _{y \in T x}\|x-y\| \leq \inf _{y \in P_{T}(x)}\|x-y\|=d\left(x, P_{T}(x)\right) .
$$

However, for $y \in P_{T}(x)$,

$$
d\left(x, P_{T}(x)\right)=\inf _{z \in P_{T}(x)}\|x-z\| \leq\|x-y\|=d(x, T x) .
$$

So we have $d(x, T x)=d\left(x, P_{T}(x)\right)$.

Since $T$ satisfies Condition $(I)$, then for $x \in K$,

$$
d\left(x, P_{T}(x)\right)=d(x, T x) \geq f\left(d(x, F(T))=f\left(d\left(x, F\left(P_{T}\right)\right) .\right.\right.
$$

That is, $P_{T}$ also satisfies Condition $(I)$.

In order to apply Theorem SZ1 to the multi-valued quasi-nonexpansive mapping $P_{T}$, we have to show that $P_{T}$ is a multi-valued mapping from $K$ to $C B(K)$.

Obviously, for each fixed $x \in K, d(x, T x)+\|x\|$ is a fixed value. Then $\forall y \in P_{T}(x)$,

$$
\|y\| \leq\|y-x\|+\|x \mid\|=d(x, T x)+\|x\|,
$$

and hence $P_{T}(x)$ is bounded.

Now, we prove that $P_{T}(x)$ is closed for any $x \in K$. In fact, for any $\left\{y_{n}\right\} \subset$ $P_{T}(x)$ with $\lim _{n \rightarrow \infty} y_{n}=y^{*}$, we have

$$
\left\|x-y_{n}\right\|=d(x, T x), \quad \lim _{n \rightarrow \infty}\left\|x-y_{n}\right\|=\left\|x-y^{*}\right\| .
$$

Thus it follows that $\left\|x-y^{*}\right\|=d(x, T x)$ and so $y^{*} \in P_{T}(x)$. This show that $P_{T}(x)$ is closed for any $x \in K$. Therefore, $P_{T}$ satisfies the requirement of Theorem SZ1 and so, the Ishikawa iterative scheme $\left\{x_{n}\right\}$ defined by $(*)$ converges to a point $x^{*} \in F\left(P_{T}\right)=F(T)$. This completes the proof.

Similarly, we also have the following which contains Theorem SZ4 as a special case.

Theorem 3. Let $K$ be a nonempty closed convex subset of a uniformly convex Banach space $E$. Suppose that $T: K \rightarrow P(K)$ is a multi-valued mapping with $F(T) \neq \emptyset$ and $P_{T}$ is continuous and quasi-nonexpansive. Assume that $T$ is hemi-compact and the sequences $\left\{\alpha_{n}\right\},\left\{\beta_{n}\right\}$ in $[0,1)$ satisfy the following conditions:

(i) $\lim _{n \rightarrow \infty} \beta_{n}=0$; 
(ii) $\sum_{n=0}^{\infty} \alpha_{n} \beta_{n}=\infty$.

Then the Ishikawa iterative scheme $\left\{x_{n}\right\}$ defined in $(*)$ converges to a fixed point of $T$.

Proof. It follows from the proof of Theorem SZ1 and Lemma 1 that

(a) for each $p \in F\left(P_{T}\right)=F(T)$, $\lim _{n \rightarrow \infty}\left\|x_{n}-p\right\|$ exists;

(b) $\lim _{n \rightarrow \infty} d\left(x_{n}, P_{T}\left(x_{n}\right)\right)=0$.

Then from the proof of Theorem 2, we have

$$
\lim _{n \rightarrow \infty} d\left(x_{n}, T x_{n}\right)=\lim _{n \rightarrow \infty} d\left(x_{n}, P_{T}\left(x_{n}\right)\right)=0 .
$$

Since $T$ is hemi-compact, there exists a subsequence $\left\{x_{n_{k}}\right\}$ of $\left\{x_{n}\right\}$ that converges to a point $x^{*} \in K$.

Now we show that $x^{*} \in F(T)$. Indeed, by the continuity of $P_{T}$, we have

$$
d\left(x^{*}, P_{T}\left(x^{*}\right)\right) \leq\left\|x^{*}-x_{n_{k}}\right\|+d\left(x_{n_{k}}, P_{T}\left(x_{n_{k}}\right)\right)+H\left(P_{T}\left(x_{n_{k}}\right), P_{T}\left(x^{*}\right)\right) \rightarrow 0 .
$$

Therefore, $x^{*} \in F\left(P_{T}\right)=F(T)$. Thus $\left\{x_{n}\right\}$ converges to $x^{*}$ since $\lim _{n \rightarrow \infty} \| x_{n}-$ $x^{*} \|$ exists by (a). This complete the proof.

Remark 1. It is easily seen that $P_{T}$ about single-valued mapping $T$ may be multi-valued. See the following example which also says $P_{T}$ isn't necessarily (quasi-)nonexpansive when $T$ is a contraction.

Example 4. Let $E=(-\infty,+\infty)$ be endowed with the Euclidean norm $\|\cdot\|=$ $|\cdot|$. Assumed that $K=[0,+\infty)$ and $T: K \rightarrow P(K)$ is defined by

$$
T x=0, \quad x \in K .
$$

Clearly, $T$ is a contraction and $F(T)=F\left(P_{T}\right)=\{0\}$. However, $P_{T}$ is not (quasi-)nonexpansive. In fact, from

$$
P_{T}(x)=\{0,2 x\}, x \in K,
$$

it follows that, for all $x, y \in K$,

$$
\begin{aligned}
H\left(P_{T}(x), P_{T}(y)\right) & =H(\{0,2 x\},\{0,2 y\}) \\
& =\max \{\min \{2 x, 2\|x-y\|\}, \min \{2 y, 2\|x-y\|\} \\
& =\left\{\begin{array}{cc}
\max \{2\|x-y\|, \min \{2 y, 2\|x-y\|\}, & x \geq y \\
\max \{\min \{2 x, 2\|x-y\|, 2\|x-y\|\}, & x \leq y
\end{array}\right. \\
& \geq\|x-y\| ;
\end{aligned}
$$

Now we give an example which is not nonexpansive but $P_{T}$ is quasi-nonexpansive. 
Example 5. Let $E=(-\infty,+\infty)$ be endowed with the Euclidean norm $\|\cdot\|=$ $|\cdot|$. Assumed that $K=[0,1]$ and $T: K \rightarrow P(K)$ is defined by

$$
T x= \begin{cases}{[0, x],} & x \in\left[0, \frac{1}{2}\right] \\ \left\{\frac{1}{2}\right\}, & x \in\left(\frac{1}{2}, 1\right] .\end{cases}
$$

Clearly, $F(T)=F\left(P_{T}\right)=\left[0, \frac{1}{2}\right]$. Since

$$
H\left(T\left(\frac{2}{5}\right), T\left(\frac{3}{5}\right)\right)=H\left(\left[0, \frac{2}{5}\right],\left\{\frac{1}{2}\right\}\right)=\frac{1}{2}>\frac{1}{5}=\left\|\frac{2}{5}-\frac{3}{5}\right\|,
$$

then $T$ is not nonexpansive. However, $P_{T}$ is quasi-nonexpansive. In fact, from

$$
P_{T}(x)= \begin{cases}\{x\}, & x \in\left[0, \frac{1}{2}\right] \\ \left\{\frac{1}{2}\right\}, & x \in\left(\frac{1}{2}, 1\right],\end{cases}
$$

it follows that, for all $x \in\left(\frac{1}{2}, 1\right]$ and $p \in\left[0, \frac{1}{2}\right]$,

$$
H\left(P_{T}(x), P_{T}(p)\right)=H\left(\left\{\frac{1}{2}\right\},\{p\}\right)=\left\|\frac{1}{2}-p\right\|<\|x-p\| .
$$

Remark 2. It is known that $P_{T}(x)=\{x\}$ for all $x \in F(T)$. The example above says that there is a multi-valued mapping $T$ that $P_{T}$ is single-valued mapping. It would be interesting to study the property of multi-valued mapping $T$ with the help of $P_{T}$.

Acknowledgments. The authors would like to thank the anonymous referees and editors for his/her valuable suggestions which helps to improve this manuscript.

\section{References}

[1] B. Panyanak, Mann and Ishikawa iterative processes for multivalued mappings in Banach spaces, Comput. Math. Appl. 54 (2007), no. 6, 872-877.

[2] K. P. R. Sastry and G. V. R. Babu, Convergence of Ishikawa iterates for a multi-valued mapping with a fixed point, Czechoslovak Math. J. 55(130) (2005), no. 4, 817-826.

[3] C. Shiau, K. K. Tan, and C. S. Wong, Quasi-nonexpansive multi-valued maps and selections, Fund. Math. 87 (1975), 109-119.

[4] N. Shahzad and H. Zegeye, On Mann and Ishikawa iteration schemes for multi-valued maps in Banach spaces, Nonlinear Anal. 71 (2009), no. 3-4, 838-844.

[5] Y. Song and H. Wang, Erratum to "Mann and Ishikawa iterative processes for multivalued mappings in Banach spaces, Comput. Math. Appl. 54 (2007), no. 6, 872-877", Comput. Math. Appl. 55 (2008), no. 12, 2999-3002.

[6] _ Convergence of iterative algorithms for multivalued mappings in Banach spaces, Nonlinear Anal. 70 (2009), no. 4, 1547-1556. 
YISHENG SONG

College of Mathematics and Information Science

Henan Normal University

XinXiang 453007, P. R. China

E-mail address: songyisheng123@yahoo.com.cn

YeOL Je Cho

Department of Mathematics Education and the RINS

Gyeongsang National University

Chinju 660-701, Korea

E-mail address: yjcho@gsnu.ac.kr 